Eur. J. Math. Anal. 2 (2022) 10

doi: 10.28924/ada/ma.2.10

\title{
Weak and Strong Convergence Theorems of Modified Projection-Type Ishikawa Iteration Scheme for Lipschitz $\alpha$-Hemicontractive Mappings
}

\author{
Imo Kalu Agwu*, Donatus Ikechi Igbokwe \\ Department of Mathematics, Micheal Okpara University of Agriculture, Umudike, Umuahia Abia State, \\ Nigeria \\ agwuimo@gmail.com,igbokwedi@yahoo.com \\ *Correspondence: agwuimo@gmail.com
}

\begin{abstract}
In this paper, we establish weak and strong convergence theorems of a two-step modified projection-type Ishikawa iterative scheme to the fixed point of $\alpha$-hemicontractive mappings without any compactness assumption on the operator or the space. Our results extend, improve and generalize several previously known results of the existing literature.
\end{abstract}

\section{INTRODUCTION}

Let $H$ be a real Hilbert space with inner product $\langle, .$,$\rangle and induced norm \|, .\|,$,$K a nonempty$ convex and closed subset of $H$ and $T: K \longrightarrow K$ a selfmap on $K$. We use $F(T)$ to denote the set of fixed point of $T, \mathbb{N}$ to denote the set of natural numbers and $x_{n} \rightarrow x$ (respectively $x_{n} \rightarrow x$ ) to denote the strong (weak) convergence of the sequence $\left\{x_{n}\right\}_{n=0}^{\infty}$ to the point $x$.

Definition 1.1. Let $T: K \longrightarrow K$ be a maaping. Then

I. $T$ is said to be $L$-Lipschitizian if there exists $L>0$ such that

$$
\|T s-T z\| \leq\|s-z\|, \forall s, z \in K
$$

From the definition, it easy to observe that every nonexpansive mapping is Lipschitizian with $L=1$.

II. $T$ is called $k$-strictly pseudocontraction (see, for example, [9]) if there exists $k \in(0,1]$ such that for all $s, z \in K$, the inequality

$$
\|T s-T z\|^{2} \leq\|s-z\|^{2}+k\|(I-T) s-(I-T) z\|^{2}
$$

hods. Note that if $k=1$ in (1.2), then $T$ is a pseudocontraction. It well-known that in real Hilbert spaces, the class of nonexpansive mapping is a proper subclass of the class of

Received: 1 Nov 2021.

Key words and phrases. strong convergence; modified Ishikawa iterative scheme; weak convergence; $\alpha$ hemicontractive operator; fixed point; real Hilbert space. 
k-strictly pseudocontive mapping. Also, the class of k-strictly pseudocontive mapping is a proper subclass of the class of pseudocontive mapping.

III. $T$ is called demicontractive mapping (see, for example, [?]) if $F(T)=\{x \in K: x=T x\} \neq \emptyset$ and $\forall(s \times q) \in(K \times F(T))$, there exists $k \in[0,1)$ such that the inequality

$$
\|T s-T q\|^{2} \leq\|s-q\|^{2}+k\|s-T s\|^{2}
$$

hods.

IV. $T$ is said to satisfy condition $A$ (see, for example [?]) $F(T)=\{x \in K: x=T x\} \neq \emptyset$ and there exists $\lambda>0$ such that

$$
\langle s-T S, s-q\rangle \geq \lambda\|s-T s\|^{2}, \forall(s \times q) \in(K \times F(T)) .
$$

It is worthy to mention that the class of $k$-strictly pseudocontions with a nonempty fixed point set is a proper subclass of the class demicontractions. $T$ is called hemicontraction (see, for example, [17]) if $k=1$ in (1.3). The class of pseudocontractive maps is a proper subclass of the class of hemicontractive maps. Again, the class of demicontractive maps is a proper subclass of the class of hemicontractive maps (see, for example, [?]). These two classes of mappings have been studied extensively by many researchers (see, for example, [?], [13], [17] and the references therein).

$V$. $T$ is called $\alpha$-demicontraction (see, for examole, [13]) if $F(T)=\{x \in K: x=T x\} \neq \emptyset$ and $\forall(s \times q) \in(K \times F(T))$, there exist $\lambda>0$ and $\alpha \geq 1$ such that the inequality

$$
\langle s-T S, s-\alpha q\rangle \geq \lambda\|s-T s\|^{2}, \forall(s \times q) \in(K \times F(T)) .
$$

holds. Clearly, (1.5) is equivalent to

$$
\|T s-\alpha q\|^{2} \leq\|s-\alpha q\|^{2}+k\|s-T s\|^{2},
$$

where $k=1-2 \lambda \in[0,1)$.

$V . T$ is called $\alpha$-hemicontraction (see, for examole, [17]) if $F(T)=\{x \in K: x=T x\} \neq \emptyset$ and $\forall(s \times q) \in(K \times F(T))$, there exists $\alpha \geq 1$ such that the inequality

$$
\|T s-\alpha q\|^{2} \leq\|s-\alpha q\|^{2}+\|s-T s\|^{2}
$$

holds. Observe that (1.7) is equivalent to

$$
\langle s-T s, s-\alpha q\rangle \geq 0, \forall(s \times q) \in(K \times F(T)) .
$$

In [[17], Example 2.2], Osilike and Onah gave an example of $\alpha$-hemicontractive mapping with $\alpha>1$ which is not hemicontractive mapping, and also showed that there are hemicontractive (1-hemicontractive) mappings which are not $\alpha$-hemicontraction for $\alpha>1$ (see [ [17], Example 2.1] for details). Again, Osilike and Onah [17] presented an example of a mapping which is hemicontractive (1-hemicontractive) and alpha-hemicontractive mapping for $\alpha>1$ but 
neither demicontractive (1-demicontractive) nor $\alpha$-demicontractive mapping for $\alpha>1$ (see [17], Example 2.3 for details). For further cheracterisation of $\alpha$-hemicontractive mapping, interested reader should consult [17].

A mapping $T: H \longrightarrow H$ is called $\nu$-strongly monotone if there exists $\nu>0$ such that

$$
\langle s-T s, s-z\rangle \geq \nu\|s-z\|^{2}, \forall s, z \in H
$$

Iterative method for approximating fixed point of $L$-Lipschitz pseudocontractive mapping has been an active area of investigation in recent times (see, for example, [?], [?], [20], [14], [26], [27] and the references contained in them). In [24], Voluhan introduced the modified projection-type Ishikawa iterative method in the following way: Let $H$ be a Hilbert space, $K$ nonempty, closed and convex subset of $H$ and $T: K \longrightarrow K$ be an L-Lipshitz pseudocontractive mapping. For an arbitrary $x 0 \in K$, define the sequence $\left\{x_{n}\right\}_{n=0}^{\infty}$ iteratively as follows.

$$
\left\{\begin{array}{l}
x_{n+1}=P_{K}\left[\left(1-\alpha_{n}-\gamma_{n}\right) x_{n}+\gamma_{n} T y_{n}\right] \\
y_{n}=\left(1-\beta_{n}\right) x_{n}+\beta_{n} T x_{n}, n \geq 1
\end{array}\right.
$$

where $\left\{\alpha_{n}\right\}_{n=0}^{\infty},\left\{\beta_{n}\right\}_{n=0}^{\infty},\left\{\gamma_{n}\right\}_{n=0}^{\infty} \in(0,1)$ and $P_{K}$ is a projection map from $H$ onto $K$. Using (1.10), she proved the following theorem.

Theorem 1.1. Let $H$ be a Hilbert space, $D$ a nonempty closed convex subset of $H$ and $T: D \longrightarrow D$ an L-Lipschitz pseudocontractive mapping such that $F(T) \neq \emptyset$. For any given $x_{0} \in H$, let $\left\{x_{n}\right\}_{n=0}^{\infty}$ be the sequence defined by (1.10). Assume the sequences $\left\{\alpha_{n}\right\}_{n=0}^{\infty},\left\{\beta_{n}\right\}_{n=0}^{\infty},\left\{\gamma_{n}\right\}_{n=0}^{\infty} \in(0,1)$ satisfy

(1) $\beta_{n}\left(1-\alpha_{n}\right)>\gamma_{n}, \forall n \geq 1$

(2) $\lim _{n \rightarrow \infty} \alpha_{n}=0$ and $\sum_{n=0}^{\infty} \alpha_{n}=\infty$;

(3) $0<\alpha \leq \gamma_{n} \leq \beta_{n} \leq \beta<\frac{1}{\sqrt{1+L^{2}}+1}, \forall n \geq 1$.

Then, the sequence $\left\{x_{n}\right\}_{n=0}^{\infty}$ strongly converges to the fixed point of $T$.

Remark 1.1. If $\alpha_{n}=0, \forall n \geq 1$, and $P_{K}$ is an identity, (1.10) reduces to the well-known Ishikawa iteration method

$$
\left\{\begin{array}{l}
x_{n+1}=\left(1-\gamma_{n}\right) x_{n}+\gamma_{n} T y_{n} \\
y_{n}=\left(1-\beta_{n}\right) x_{n}+\beta_{n} T x_{n}, n \geq 1
\end{array}\right.
$$

which has been used by several researchers to approximate the fixed points of different operators or operator equations in different spaces.

Motivated and inspired by the works in [17], [24] and some ongoing research in this direction, it is our purpose in this paper to extend the results in [24] and other related results from Lipschitz pseudocontractive mapping to the more general $\alpha$-hemicontractive mapping. Our results is more 
general and also more applicable because fewer and simpler conditions are required to attain convergence.

\section{Preliminary}

The following definitionS and lemmas will be needed to prove our main results.

Definition 2.1. (see [27]) Let $H$ and $K$ be as defined above. For each $x \in H$, there exists $a$ unique nearest point of $K$, denoted by $P_{K} X$, such that

$$
\left\|x-P_{K} x\right\| \leq\|x-y\|, \forall y \in K .
$$

Such a $P_{K}$ is called metric projection from $H$ onto $K$. It is well-known that $P_{K}$ is firmly nonexpansive mapping from $H$ onto $K$; that is,

$$
\left\|P_{K} x-P_{K} y\right\|^{2} \leq\left\langle P_{K} x-P_{K} y, x-y\right\rangle, \forall x, y \in H .
$$

Also, for any $x \in H$ and $z \in K, z=P_{K} x$ if and only if

$$
\langle x-z, z-y\rangle \geq 0, \forall y \in K
$$

Definition 2.2. The Banach space $Z$ is said to have Opial property, if for each weakly convergent sequence $\left\{z_{n}\right\}_{n=0}^{\infty}$ with weak limit $z \in Z$, the following inequality holds:

$$
\limsup _{n \rightarrow \infty}\left\|z_{n}-z\right\|<\left\|z_{n}-y\right\|, \forall y \in Z \text { withz } \neq y .
$$

Note that all finite dimensional Banach spaces, all Hilbert spaces and $\ell^{p}(0 \leq p<\infty)$ satisfy the Opial property. But $L_{p}(1<p<\infty . p \neq 2)$ do not satisfies the Opial property.

Definition 2.3. (see [27]) Let $E$ be a real Banach space. A mapping $T$, with domain $D(T) \in E$, is said to be demiclosed at 0 if for any sequence $z_{n} \subset E, z_{n} \uparrow q \in D(T)$ and $\left\|z_{n}-T z_{n}\right\| \rightarrow 0$, then $T q=q$.

Lemma 2.1. (see [27]) Let $H$ be a real Hilbert space. Then, the following inequality holds:

$$
\|\lambda x+(1-\lambda) y\|^{2} \leq \lambda\|x\|^{2}+(1-\lambda)\|y\|^{2}-\lambda(1-\lambda)\|x-y\|, \forall \lambda \in[0,1], \forall x, y \in H .
$$

Lemma 2.2. (see [27]) Let $\left\{s_{n}\right\}_{n \in N}$ be a sequence of nonnegative real numbers satisfying the inequality:

$$
s_{n+1} \leq\left(1-\gamma_{n}\right) s_{n}+\delta_{n}, \forall n \geq 1
$$

where $\left\{\gamma_{n}\right\}_{n \in N}$ and $\left\{\delta_{n}\right\}_{n \in N}$ satisfy the following conditions:

(i) $\left\{\gamma_{n}\right\}_{n \in N} \subset(0,1)$;

(ii) $\sum_{n=1}^{\infty} \gamma_{n}=\infty$.

Suppose $\sum_{n=1}^{\infty} \delta_{n}<\infty$, then, $\lim _{n \rightarrow \infty} s_{n}=0$. 
Lemma 2.3. (see [4]) Let $E$ be a real Hilbert space. Then, for all $x, y \in H$, the following inequalities hold:

$$
\begin{aligned}
& \text { I. }\|x-y\|^{2} \leq\|x\|^{2}-2\langle y,(x+y)\rangle+\|y\|^{2} \\
& \text { II. }\|x-y\|^{2} \leq\|x\|^{2}-2\langle y,(x+y)\rangle .
\end{aligned}
$$

Lemma 2.4. (see [?]) Let $D$ be a sunset of a real Hilbert space, $T: D \longrightarrow H$ be a nonexpansive mapping and $z$ a weak cluster point of the sequence $\left\{y_{n}\right\}_{n=0}^{\infty}$. If $\left\|T y_{n}-y_{n}\right\| \rightarrow 0$, then $z \in F(T)$

Proposition 2.5. (see [27]) Let $D$ be a nonempty subset of a real Hilbert space amd $\Gamma: D \longrightarrow D$ an $\alpha$-demicontractive mapping. Assume that $x \in D$ and $\alpha \geq 1$. Then, $\Gamma$ is Lipschitizian.

Theorem 2.6. (see [4]) A Banach space $E$ is reflexive if and only if every (normed) bounded sequence in $E$ has a subsequence which converges weakly to an element of $E$.

\section{Convergence Results}

Now, we prove our main results.

Theorem 3.1. Let $H$ be a real Hilbert space, $K$ a nonempty closed convex subset of $H$ and $T$ : $K \longrightarrow K$ an L-Lipschitz $\alpha$-hemicontractive mapping. For any arbitrary $x_{0} \in H$, define the sequence $\left\{x_{n}\right\}_{n=0}^{\infty}$ iteratively as follows:

$$
\left\{\begin{array}{l}
x_{n+1}=P_{K}\left[\left(1-\alpha_{n}-\gamma_{n}\right) x_{n}+\gamma_{n} T y_{n}\right] \\
y_{n}=\left(1-\beta_{n}\right) x_{n}+\beta_{n} T x_{n}, n \geq 1
\end{array}\right.
$$

where the sequences $\left\{\delta_{n}\right\}_{n=0}^{\infty},\left\{\gamma_{n}\right\}_{n=0}^{\infty},\left\{\beta_{n}\right\}_{n=0}^{\infty} \in(0,1)$ satisfy the following conditions:

(i) $0<\delta \leq \delta_{n} \leq \beta_{n} \leq \gamma_{n} \leq \gamma \leq \frac{1-\delta}{1+L^{2}}$;

(ii) $\lim _{n \rightarrow \infty} \delta_{n}=0$ and $\sum_{n=0}^{\infty} \delta_{n}=\infty$.

Then, the sequence $\left\{x_{n}\right\}_{n=0}^{\infty}$ generated by (3.1) weakly and strongly converges to the fixed point of $T$.

Proof. Since $F(T)$ is nonempty, let $\alpha q \in F(T)$ and $x \in K$. Using (3.1), Lemma 2.1 and the fact that $T$ is $L$-Lipschitizian, we estimate as follows:

$$
\begin{aligned}
\left\|x_{n+1}-\alpha q\right\|^{2} & =\left\|P_{K}\left[\left(1-\delta_{n}-\gamma_{n}\right) x_{n}+\gamma_{n} T y_{n}\right]-\alpha q\right\| \\
& \leq\left\|\left(1-\delta_{n}-\gamma_{n}\right) x_{n}+\gamma_{n} T y_{n}-\alpha q\right\| \\
& =\left\|\left(1-\delta_{n}-\gamma_{n}\right)\left(x_{n}-\alpha q\right)+\gamma_{n}\left(T y_{n}-\alpha q\right)-\delta_{n} \alpha q\right\| \\
& \leq\left\|\left(1-\delta_{n}-\gamma_{n}\right)\left(x_{n}-\alpha q\right)+\gamma_{n}\left(T y_{n}-\alpha q\right)\right\|+\delta_{n}\|\alpha q\| .
\end{aligned}
$$

Set $Q_{n}=\left\|\left(1-\delta_{n}-\gamma_{n}\right)\left(x_{n}-\alpha q\right)+\gamma_{n}\left(T y_{n}-\alpha q\right)\right\|^{2}$ and observe that

$$
Q_{n}=\left\|\left(1-\delta_{n}\right)\left(x_{n}-\alpha q\right)-\left(1-\gamma_{n}\right)\left(x_{n}-\alpha q\right)+\gamma_{n}\left(T y_{n}-\alpha q\right)\right\|^{2} .
$$


Since

$$
\left.\left(1-\delta_{n}\right)\left(x_{n}-\alpha q\right)=\left(1-\delta_{n}\right)\left(1-\gamma_{n}\right)\left(x_{n}-\alpha q\right)+\gamma_{n}\left(1-\delta_{n}\right)\right)\left(x_{n}-\alpha q\right)
$$

and

$$
\gamma_{n}\left(T y_{n}-\alpha q\right)=\gamma_{n}\left(1-\delta_{n}\right)\left(T y_{n}-\alpha q\right)+\gamma_{n} \delta_{n}\left(T y_{n}-\alpha q\right)
$$

it follows from (3.3) that

$$
\begin{aligned}
Q_{n}= & \left.\|\left(1-\delta_{n}\right)\left(1-\gamma_{n}\right)\left(x_{n}-\alpha q\right)+\gamma_{n}\left(1-\delta_{n}\right)\right)\left(x_{n}-\alpha q\right)-\left(1-\gamma_{n}\right)\left(x_{n}-\alpha q\right) \\
& +\gamma_{n}\left(1-\delta_{n}\right)\left(T y_{n}-\alpha q\right)+\gamma_{n} \delta_{n}\left(T y_{n}-\alpha q\right) \|^{2} \\
= & \left\|\left(1-\delta_{n}\right)\left[\left(1-\gamma_{n}\right)\left(x_{n}-\alpha q\right)+\gamma_{n}\left(T y_{n}-\alpha q\right)\right]+\delta_{n} \gamma_{n}\left(T y_{n}-x_{n}\right)\right\|^{2} .
\end{aligned}
$$

(3.6) and Lemma 2.1 imply that

$$
\begin{aligned}
Q_{n}= & \left(1-\delta_{n}\right)\left\|\left(1-\gamma_{n}\right)\left(x_{n}-\alpha q\right)+\gamma_{n}\left(T y_{n}-\alpha q\right)\right\|^{2}+\delta_{n}\left\|\gamma_{n}\left(T y_{n}-x_{n}\right)\right\|^{2} \\
& -\delta_{n}\left(1-\delta_{n}\right)\left\|x_{n}-\alpha q\right\|^{2} .
\end{aligned}
$$

If we denote $V_{n}=\left\|\left(1-\gamma_{n}\right)\left(x_{n}-\alpha q\right)+\gamma_{n}\left(T y_{n}-\alpha q\right)\right\|^{2}$ and use similar technique as above, then we get

$$
V_{n}=\left(1-\gamma_{n}\right)\left\|x_{n}-\alpha q\right\|^{2}+\gamma_{n}\left\|T y_{n}-\alpha q\right\|^{2}-\gamma_{n}\left(1-\gamma_{n}\right)\left\|x_{n}-T y_{n}\right\|^{2} .
$$

(3.7) and (3.8) imply

$$
\begin{aligned}
Q_{n}= & \left(1-\delta_{n}\right)\left[\left(1-\gamma_{n}\right)\left\|x_{n}-\alpha q\right\|^{2}+\gamma_{n}\left\|T y_{n}-\alpha q\right\|^{2}-\gamma_{n}\left(1-\gamma_{n}\right)\left\|x_{n}-T y_{n}\right\|^{2}\right] \\
& +\delta_{n} \gamma_{n}^{2}\left\|T y_{n}-x_{n}\right\|^{2}-\delta_{n}\left(1-\delta_{n}\right)\left\|x_{n}-\alpha q\right\|^{2} \\
= & \left(1-\delta_{n}\right)\left(1-\gamma_{n}\right)\left\|x_{n}-\alpha q\right\|^{2}+\left(1-\delta_{n}\right) \gamma_{n}\left\|T y_{n}-\alpha q\right\|^{2}-\gamma_{n}\left(1-\gamma_{n}\right)\left(1-\delta_{n}\right)\left\|x_{n}-T y_{n}\right\|^{2} \\
& +\delta_{n} \gamma_{n}^{2}\left\|T y_{n}-x_{n}\right\|^{2}-\delta_{n}\left(1-\delta_{n}\right)\left\|x_{n}-\alpha q\right\|^{2} \\
\leq & \left(1-\delta_{n}\right)\left(1-\gamma_{n}\right)\left\|x_{n}-\alpha q\right\|^{2}+\left(1-\delta_{n}\right) \gamma_{n} L^{2}\left\|y_{n}-\alpha q\right\|^{2} \\
& -\left(\gamma_{n}-\delta_{n} \gamma_{n}-\gamma_{n}^{2}+\gamma_{n}^{2} \delta_{n}\right)\left\|x_{n}-T y_{n}\right\|^{2}+\delta_{n} \gamma_{n}^{2}\left\|T y_{n}-x_{n}\right\|^{2}-\delta_{n}\left(1-\delta_{n}\right)\left\|x_{n}-\alpha q\right\|^{2} \\
= & \left(1-\delta_{n}\right)\left(1-\gamma_{n}\right)\left\|x_{n}-\alpha q\right\|^{2}+\gamma_{n} L^{2}\left\|y_{n}-\alpha q\right\|^{2}-\delta_{n} \gamma_{n} L^{2}\left\|y_{n}-\alpha q\right\|^{2} \\
& -\left(\gamma_{n}-\delta_{n} \gamma_{n}-\gamma_{n}^{2}\right)\left\|x_{n}-T y_{n}\right\|^{2}-\delta_{n}\left(1-\delta_{n}\right)\left\|x_{n}-\alpha q\right\|^{2} .
\end{aligned}
$$

Observr that

$$
\begin{aligned}
\mid x_{n}-T y_{n} \| & \leq\left(\left\|x_{n}-\alpha q\right\|+L\left\|y_{n}-\alpha q\right\|\right)^{2} \\
& =\left\|x_{n}-\alpha q\right\|^{2}+L\left(2\left\|x_{n}-\alpha q\right\|\left\|y_{n}-\alpha q\right\|\right)+L^{2}\left\|y_{n}-\alpha q\right\|^{2} \\
& \leq\left\|x_{n}-\alpha q\right\|^{2}+L\left\|x_{n}-\alpha q\right\|^{2}+L\left\|y_{n}-\alpha q\right\|^{2}+L^{2}\left\|y_{n}-\alpha q\right\|^{2} \\
& =(1+L)\left\|x_{n}-\alpha q\right\|^{2}+L(1+L)\left\|y_{n}-\alpha q\right\|^{2} .
\end{aligned}
$$


(3.9) and (3.10) imply

$$
\begin{aligned}
Q_{n} \leq & \left(1-\delta_{n}\right)\left(1-\gamma_{n}\right)\left\|x_{n}-\alpha q\right\|^{2}+\gamma_{n} L^{2}\left\|y_{n}-\alpha q\right\|^{2}-\delta_{n} \gamma_{n} L^{2}\left\|y_{n}-\alpha q\right\|^{2} \\
& -\left(\gamma_{n}-\delta_{n} \gamma_{n}-\gamma_{n}^{2}\right)\left[(1+L)\left\|x_{n}-\alpha q\right\|^{2}+L(1+L)\left\|y_{n}-\alpha q\right\|^{2}\right]-\delta_{n}\left(1-\delta_{n}\right)\left\|x_{n}-\alpha q\right\|^{2} \\
= & \left(1-\delta_{n}\right)\left(1-\gamma_{n}\right)\left\|x_{n}-\alpha q\right\|^{2}-(1+L)\left(\gamma_{n}-\delta_{n} \gamma_{n}-\gamma_{n}^{2}\right)\left\|x_{n}-\alpha q\right\| \\
& -\left[\left(\gamma_{n}-\delta_{n} \gamma_{n}-\gamma_{n}^{2}\right) L-L^{2} \gamma_{n}^{2}\right]\left\|y_{n}-\alpha q\right\|^{2}-\delta_{n}\left(1-\delta_{n}\right)\left\|x_{n}-\alpha q\right\|^{2}
\end{aligned}
$$

Again, from (3.1), we get

$$
\left\|y_{n}-\alpha q\right\|^{2}=\left\|\left(1-\beta_{n}\right)\left(x_{n}-\alpha q\right)+\beta_{n}\left(T x_{n}-\alpha q\right)\right\|^{2}
$$

Since $T$ is $\alpha$-hemicontractive mapping, it follows from (3.12) and Lemma 2.1 that

$$
\begin{aligned}
\left\|y_{n}-\alpha q\right\|^{2} & \leq\left(1-\beta_{n}\right)\left\|x_{n}-\alpha q\right\|^{2}+\beta_{n}\left[\left\|x_{n}-\alpha q\right\|^{2}\left\|^{2}+\right\| x_{n}-T x_{n} \|^{2}\right]-\beta_{n}\left(1-\beta_{n}\right)\left\|x_{n}-T x_{n}\right\|^{2} \\
& =\left(1-\beta_{n}\right)\left\|x_{n}-\alpha q\right\|^{2}+\beta_{n}^{2}\left\|x_{n}-T x_{n}\right\|^{2}
\end{aligned}
$$

Putting (3.13) into (3.11), we have

$$
\begin{aligned}
Q_{n} \leq & \left(1-\delta_{n}\right)\left(1-\gamma_{n}\right)\left\|x_{n}-\alpha q\right\|^{2}-(1+L)\left(\gamma_{n}-\delta_{n} \gamma_{n}-\gamma_{n}^{2}\right)\left\|x_{n}-\alpha q\right\| \\
& -\left[\left(\gamma_{n}-\delta_{n} \gamma_{n}-\gamma_{n}^{2}\right) L-L^{2} \gamma_{n}^{2}\right]\left\{\left(1-\beta_{n}\right)\left\|x_{n}-\alpha q\right\|^{2}+\beta_{n}^{2}\left\|x_{n}-T x_{n}\right\|^{2}\right\} \\
& -\delta_{n}\left(1-\delta_{n}\right)\left\|x_{n}-\alpha q\right\|^{2} \\
\leq & \left(1-\delta_{n}\right)\left(1-\gamma_{n}\right)\left\|x_{n}-\alpha q\right\|^{2}-\left[\left(\gamma_{n}-\delta_{n} \gamma_{n}-\gamma_{n}^{2}\right)(1+L)+\delta_{n}\left(1-\delta_{n}\right)-L^{2} \gamma_{n}^{2}\right]\left\|x_{n}-\alpha q\right\|^{2} \\
& -\beta_{n}^{2}\left[\left(\gamma_{n}-\delta_{n} \gamma_{n}-\gamma_{n}^{2}\right) L-L^{2} \gamma_{n}^{2}\right]\left\|x_{n}-T x_{n}\right\|^{2} .
\end{aligned}
$$

Since from condition (i), $\left(\gamma_{n}-\delta_{n} \gamma_{n}-\gamma_{n}^{2}\right)-L^{2} \gamma_{n}^{2} \geq 0$, it follows from (3.14) that

$$
Q_{n} \leq\left(1-\delta_{n}\right)^{2}\left\|x_{n}-\alpha q\right\|^{2}
$$

(3.2) and (3.15) imply

$$
\begin{aligned}
\mid x_{n+1}-\alpha q \| & \leq\left(1-\delta_{n}\right)\left\|x_{n}-\alpha q\right\|^{2}+\delta_{n}\|\alpha q\| \\
& \leq \max \left\{\left\|x_{n}-\alpha q\right\|^{2},\|\alpha q\|\right\}, \forall n \in \mathbb{N}
\end{aligned}
$$

It is easy to see, using mathematical induction, that

$$
\begin{aligned}
\mid x_{n+1}-\alpha q \| & \leq \max \left\{\left\|x_{n}-\alpha q\right\|^{2},\|\alpha q\|\right\} \\
& =\left\|x_{0}-\alpha q\right\|^{2} .
\end{aligned}
$$


Hence, $\left\{x_{n}\right\}_{n=0}^{\infty}$ is bounded.

Furthermore, since from (3.1),

$$
\begin{aligned}
\left\|x_{n+1}-\alpha q\right\|^{2} & =\left\|P_{K}\left[\left(1-\delta_{n}-\gamma_{n}\right) x_{n}+\gamma_{n} T y_{n}\right]-\alpha q\right\|^{2} \\
& \leq\left\|\left(1-\delta_{n}-\gamma_{n}\right) x_{n}+\gamma_{n} T y_{n}-\alpha q\right\|^{2} \\
& =\left\|x_{n}-\alpha q-\gamma_{n}\left(x_{n}-T y_{n}\right)-\delta_{n} x_{n}\right\|^{2}
\end{aligned}
$$

it follows from Lemma 2.3(i) that

$$
\left\|x_{n+1}-\alpha q\right\|^{2} \leq\left\|x_{n}-\alpha q-\gamma_{n}\left(x_{n}-T y_{n}\right)\right\|^{2}-2 \delta_{n}\left\langle x_{n}, x_{n+1}-\alpha q\right\rangle .
$$

Since

$$
\begin{aligned}
\left\|x_{n}-\alpha q-\gamma_{n}\left(x_{n}-T y_{n}\right)\right\|^{2}= & \left\|\left(1-\gamma_{n}\right)\left(x_{n}-\alpha q\right)+\gamma_{n}\left(\alpha q-T y_{n}\right)\right\|^{2} \\
= & \left(1-\gamma_{n}\right)\left\|x_{n}-\alpha q\right\|^{2}+\gamma_{n}\left\|\alpha q-T y_{n}\right\|^{2}-\gamma_{n}\left(1-\gamma_{n}\right)\left\|T y_{n}-x_{n}\right\|^{2} \\
\leq & \left(1-\gamma_{n}\right)\left\|x_{n}-\alpha q\right\|^{2}+\gamma_{n} L^{2}\left\|y_{n}-\alpha q\right\|^{2} \\
& -\gamma_{n}\left(1-\gamma_{n}\right)\left\|T y_{n}-x_{n}\right\|^{2},
\end{aligned}
$$

it follows from (3.10) that

$$
\begin{aligned}
\left\|x_{n}-\alpha q-\gamma_{n}\left(x_{n}-T y_{n}\right)\right\|^{2} \leq & \left(1-\gamma_{n}\right)\left\|x_{n}-\alpha q\right\|^{2}+\gamma_{n} L^{2}\left\|y_{n}-\alpha q\right\|^{2} \\
& -\gamma_{n}\left(1-\gamma_{n}\right)\left\{(1+L)\left\|x_{n}-\alpha q\right\|^{2}+L(1+L)\left\|y_{n}-\alpha q\right\|^{2}\right\} \\
= & \left(1-\gamma_{n}\right)\left\|x_{n}-\alpha q\right\|^{2}+\gamma_{n} L^{2}\left\|y_{n}-\alpha q\right\|^{2} \\
& -\gamma_{n}\left(1-\gamma_{n}\right)(1+L)\left\|x_{n}-\alpha q\right\|^{2}-\gamma_{n}\left(1-\gamma_{n}\right) L\left\|y_{n}-\alpha q\right\|^{2} \\
& -\gamma_{n} L^{2}\left\|y_{n}-\alpha q\right\|^{2}+\gamma_{n}^{2} L^{2}\left\|y_{n}-\alpha q\right\|^{2} \\
= & \left(1-\gamma_{n}\right)\left\|x_{n}-\alpha q\right\|^{2}-\gamma_{n}\left(1-\gamma_{n}\right)(1+L)\left\|x_{n}-\alpha q\right\|^{2} \\
& -\left[\gamma_{n}\left(1-\gamma_{n}\right) L-L^{2} \gamma_{n}^{2}\right]\left\|y_{n}-\alpha q\right\|^{2} .
\end{aligned}
$$

(3.13) and (3.19) imply

$$
\begin{aligned}
\left\|x_{n}-\alpha q-\gamma_{n}\left(x_{n}-T y_{n}\right)\right\|^{2} \leq & \left(1-\gamma_{n}\right)\left\|x_{n}-\alpha q\right\|^{2}-\gamma_{n}\left(1-\gamma_{n}\right)(1+L)\left\|x_{n}-\alpha q\right\|^{2} \\
& -\left[\gamma_{n}\left(1-\gamma_{n}\right) L-L^{2} \gamma_{n}^{2}\right]\left\{\left(1-\beta_{n}\right)\left\|x_{n}-\alpha q\right\|^{2}+\beta_{n}^{2}\left\|x_{n}-T x_{n}\right\|^{2}\right\} \\
\leq & \left(1-\gamma_{n}\right)\left\|x_{n}-\alpha q\right\|^{2}-\gamma_{n} L\left[1-\gamma_{n}-\gamma_{n} L\right]\left\{\left(1-\beta_{n}\right)\left\|x_{n}-\alpha q\right\|^{2}\right. \\
& \left.+\beta_{n}^{2}\left\|x_{n}-T x_{n}\right\|^{2}\right\} .
\end{aligned}
$$

By condition (i), $1-\gamma_{n}-\gamma_{n} L>0, \forall n \geq 0$. Consequently,

$$
\begin{aligned}
\left\|x_{n}-\alpha q-\gamma_{n}\left(x_{n}-T y_{n}\right)\right\|^{2} \leq & \left\|x_{n}-\alpha q\right\|^{2} \\
& -\left(1-\gamma_{n}-\gamma_{n} L\right) \beta_{n}^{2} \gamma_{n} L\left\|x_{n}-T x_{n}\right\|^{2} .
\end{aligned}
$$


(3.17)and (3.21) imply

$$
\begin{aligned}
\left\|x_{n+1}-\alpha q\right\|^{2} \leq & \left\|x_{n}-\alpha q\right\|^{2}-\left(1-\gamma_{n}-\gamma_{n} L\right) \beta_{n}^{2} \gamma_{n} L\left\|x_{n}-T x_{n}\right\|^{2} \\
& -2 \delta_{n}\left\langle x_{n}, x_{n+1}-\alpha q\right\rangle .
\end{aligned}
$$

Since $\left\{x_{n}\right\}$ is bounded, there exists a constant $B>0$ such that $-2\left\langle x_{n}, x_{n+1}-\alpha q\right\rangle \leq B$. Thus,

$$
\begin{aligned}
\left\|x_{n+1}-\alpha q\right\|^{2} \leq & \left\|x_{n}-\alpha q\right\|^{2}-\left(1-\gamma_{n}-\gamma_{n} L\right) \beta_{n}^{2} \gamma_{n} L\left\|x_{n}-T x_{n}\right\|^{2} \\
& \delta_{n} B .
\end{aligned}
$$

The last inequality implies that

$$
\left\|x_{n+1}-\alpha q\right\|^{2}-\left\|x_{n}-\alpha q\right\|^{2}+\left(1-\gamma_{n}-\gamma_{n} L\right) \beta_{n}^{2} \gamma_{n} L\left\|x_{n}-T x_{n}\right\|^{2} \leq \delta_{n} B .
$$

Now, we consider the following two cases:

Case A: Suppose there exists $n_{0} \in \mathbb{N}$ such that $\left\{\left\|x_{n}-\alpha q\right\|\right\}$ is non-increasing. Then, $\left\{\left\|x_{n}-\alpha q\right\|\right\}$ is convergent. Clearly, $\left\|x_{n+1}-\alpha q\right\|-\left\|x_{n}-\alpha q\right\| \rightarrow 0$. In view, of condition (ii) and (3.22), we have $\left\|x_{n}-T x_{n}\right\| \rightarrow 0$. By Lemma 2.4, it is obvious that $\omega_{\omega}\left(x_{n}\right) \subset F(T)$, where $\omega_{\omega}\left(x_{n}\right)\left\{x: \exists x_{n_{k}} \rightarrow \alpha x^{\star}\right\}$ is the weak limit set of $\left\{x_{n}\right\}$. This implies that the sequence $\left\{x_{n}\right\}$ converges weakly to a fixed point $\alpha x^{\star}$ of $T$.

Suppose there exists some subsequences $\left\{x_{n_{k}}\right\}_{k=0}^{\infty} \subset\left\{x_{n}\right\}_{n=0}^{\infty}$ such that $x_{n_{k}} \rightarrow \alpha y^{\star}$ weakly and $\alpha y^{\star} \neq \alpha x^{\star}$. Since $\lim _{n \rightarrow \infty}\left\|x_{n}-\alpha v\right\|$ exists for $\alpha v \in F(T)$, by virtue of Opial condition on $H$, we have

$$
\begin{aligned}
\lim _{n \rightarrow \infty}\left\|x_{n}-\alpha x^{\star}\right\| & =\lim _{n \rightarrow \infty}\left\|x_{n_{j}}-\alpha x^{\star}\right\|<\lim _{n \rightarrow \infty}\left\|x_{n_{j}}-\alpha y^{\star}\right\|=\lim _{n \rightarrow \infty}\left\|x_{n_{k}}-\alpha y^{\star}\right\| \\
& <\lim _{n \rightarrow \infty}\left\|x_{n_{k}}-\alpha x^{\star}\right\|=\lim _{n \rightarrow \infty}\left\|x_{n_{j}}-\alpha y^{\star}\right\|,
\end{aligned}
$$

which is a contradiction. Consequently, $\alpha y^{\star}=\alpha x^{\star}$. This implies that $\left\{x_{n_{j}}\right\}_{j=0}^{\infty}$ converges wealy to a common fixed point of $T$.

Next, we prove that $\left\{x_{n}\right\}_{n=0}^{\infty}$ converges strongly to $x^{\star /}$ Let $\xi_{n}=\gamma_{n} T y_{n}+\left(1-\gamma_{n} x_{n}\right)$. Then, from (3.1), we obtain $x_{n+1}=P_{K}\left[\xi_{n}-\delta_{n} x_{n}\right], n \geq 0$. This implies that

$$
\begin{aligned}
x_{n+1} & =P_{K}\left[\xi_{n}+\delta_{n} \xi_{n}+\delta_{n} \xi_{n}-\delta_{n} x_{n}\right. \\
& =P_{K}\left[\left(1-\delta_{n}\right) \xi_{n}+\delta_{n}\left(\xi_{n}-x_{n}\right)\right] .
\end{aligned}
$$

Observe that

$$
\left\|\xi_{n}-\alpha x^{\star}\right\|^{2}=\left\|x_{n}-\alpha x^{\star}-\gamma_{n}\left(x_{n}-T y_{n}\right)\right\|^{2} .
$$

By using the same argument as in (3.20), with $\alpha x^{\star}=\alpha$, we get, from (3.24), that

$$
\left\|\xi_{n}-\alpha x^{\star}\right\|=\left\|x_{n}-\alpha x^{\star}\right\| .
$$

Again, from (3.1), we obtain

$$
\left\|y_{n}-x_{n}\right\|=\beta_{n}\left\|x_{n}-T x_{n}\right\| \rightarrow 0 \text { as } n \rightarrow \infty, \beta_{n} \in(0,1) .
$$


In addition, since $T$ is Lipschitz, it follows that

$$
\begin{aligned}
\left\|\xi_{n}-x_{n}\right\| & =\left\|\gamma_{n}\left[\left(T y_{n}-T x_{n}\right)-\left(x_{n}-T x_{n}\right)\right]\right\| \\
& \leq \gamma_{n}\left\|T y_{n}-T x_{n}\right\|-\gamma_{n}\left\|x_{n}-T x_{n}\right\| \\
& \leq \gamma_{n} L\left\|y_{n}-x_{n}\right\|+\gamma_{n}\left\|x_{n}-T x_{n}\right\| \rightarrow 0 \text { as } n \rightarrow \infty
\end{aligned}
$$

Now, using (3.23), we get

$$
\begin{aligned}
\left\|x_{n+1}-\alpha x^{\star}\right\|^{2} & \leq\left\|\left(1-\delta_{n}\right) \xi_{n}+\delta_{n}\left(\xi_{n}-x_{n}\right)-\alpha x^{\star}\right\|^{2} \\
& =\left\|\left(1-\delta_{n}\right)\left(\xi_{n}-\alpha x^{\star}\right)+\delta_{n}\left(\xi_{n}-x_{n}\right)-\delta_{n} \alpha x^{\star}\right\|^{2},
\end{aligned}
$$

which by Lemma 2.3 yields

$$
\begin{aligned}
\left\|x_{n+1}-\alpha x^{\star}\right\|^{2} \leq & \left\|\left(1-\delta_{n}\right)\left(\xi_{n}-\alpha x^{\star}\right)+\delta_{n}\left(\xi_{n}-x_{n}\right)\right\|^{2}-2 \delta_{n}\left\langle\alpha x^{\star}, x_{n+1}-\alpha x^{\star}\right\rangle \\
= & \left(1-\delta_{n}\right)\left\|\xi_{n}-\alpha x^{\star}\right\|^{2}+\delta_{n}\left\|\xi_{n}-x_{n}\right\|^{2}-\delta_{n}\left(1-\delta_{n}\right)\left\|x_{n}-\alpha x^{\star}\right\|^{2} \\
& -2 \delta_{n}\left\langle\alpha x^{\star}, x_{n+1}-\alpha x^{\star}\right\rangle \\
\leq & \left(1-\delta_{n}\right)\left\|\xi_{n}-\alpha x^{\star}\right\|^{2}+\left\|\xi_{n}-x_{n}\right\|^{2}-2 \delta_{n}\left\langle\alpha x^{\star}, x_{n+1}-\alpha x^{\star}\right\rangle \\
= & \left(1-\delta_{n}\right)\left\|\xi_{n}-\alpha x^{\star}\right\|^{2}-2 \delta_{n}\left\langle\alpha x^{\star}, x_{n+1}-\alpha x^{\star}\right\rangle \quad(\text { by (3.27)) } \\
\leq & \left(1-\delta_{n}\right)\left\|\xi_{n}-\alpha x^{\star}\right\|^{2}
\end{aligned}
$$

(3.29) and Lemma 2.2 imply that $x_{n} \rightarrow \alpha x^{\star}$ as $n \rightarrow \infty$.

Case B: Assume that $\left\{\left\|x_{n}-\alpha q\right\|\right\}_{n=0}^{\infty}$ is not a monotonically increasing sequence. Set $V_{n}=$ $\left\|x_{n}-\alpha q\right\|^{2}$ and let $\tau: \mathbb{N} \longrightarrow \mathbb{N}$ be a mapping defined by

$$
\tau_{n}=\max \left\{k \in \mathbb{N}: k \leq n, V_{n} \leq V_{n+1}\right\}, \forall n \geq n_{0},
$$

for some $n_{0}$ large enough. Obviously, $\left\{\tau_{n}\right\}_{n=0}^{\infty}$ is a nondecreasing sequence given that $\tau_{n} \rightarrow$ $\infty$ as $n \rightarrow \infty$ and $V_{\tau_{n}} \leq V_{\tau_{n+1}}$ for all $n \geq n_{0}$. From (3.22),

$$
\left\|x_{\tau(n)}-T x_{\tau(n)}\right\|^{2} \leq \frac{\delta_{\tau(n)} B}{\left(1-\gamma_{\tau(n)}-\gamma_{\tau(n)} L\right) \beta_{\tau(n)}^{2} \gamma_{\tau(n)} L} \rightarrow 0 \text { as } n \rightarrow \infty .
$$

Therefore, $\lim _{n \rightarrow \infty}\left\|x_{\mathcal{T}(n)}-T x_{\mathcal{\tau}(n)}\right\|=0$. Using similar argument as Case $\mathrm{A}$ above, we conclude that $\left\{x_{\tau(n)}\right\} \rightarrow \alpha x^{\star} \rightarrow \infty$.

From (3.28), we have

$$
0 \leq\left\|x_{\tau(n)+1}-\alpha x^{\star}\right\|^{2}-\left\|x_{\tau(n)}-\alpha x^{\star}\right\|^{2} \leq \delta_{\tau(n)}\left[2\left\langle\alpha x^{\star}-x_{\tau(n)+1}-\left\|x_{\tau(n)}-\alpha x^{\star}\right\|^{2}\right],\right.
$$

for $\delta_{\tau(n)} \in(0,1)$. Hence, $\lim _{n \rightarrow \infty}\left\|x_{\tau(n)}-\alpha x^{\star}\right\|^{2}=0$. This implies that $\lim _{n \rightarrow \infty} V_{\tau(n)}=$ $\lim _{n \rightarrow \infty} V_{\tau(n)+1}=0$. In addition, for $n \geq n_{0}$, it is easy to see that $V_{\tau(n)}=V_{\tau(n)+1}$ if $n \neq \tau(n)$ (i.e., $\tau(n)<n$ ) because $V_{j}>V_{j+1}$, for $\tau(n)+1 \leq n$. Consequently. we obtain, for all $n \geq n_{0}$, $0 \leq V_{\tau(n)} \max \left\{V_{\tau(n)}, V_{\tau(n)+1}\right\}=V_{\tau(n)+1}$. Hence, $\lim _{n \rightarrow \infty} V_{n}=0$. That is, $\left\{x_{n}\right\}_{n=0}^{\infty}$ converges 
strongly to $\alpha x^{\star}$, and this completes the proof.

The following corollaries are immediate consequence of Theorem 3.1.

Corollary 3.2. Let $H$ be a real Hilbert space, $K$ a nonempty closed convex subset of $H$ and $T: K \longrightarrow K$ an L-Lipschitz hemicontractive mapping. For any arbitrary $x_{0} \in H$, define the sequence $\left\{x_{n}\right\}_{n=0}^{\infty}$ iteratively as follows:

$$
\left\{\begin{array}{l}
x_{n+1}=P_{K}\left[\left(1-\alpha_{n}-\gamma_{n}\right) x_{n}+\gamma_{n} T y_{n}\right] \\
y_{n}=\left(1-\beta_{n}\right) x_{n}+\beta_{n} T x_{n}, n \geq 1
\end{array}\right.
$$

where the sequences $\left\{\delta_{n}\right\}_{n=0}^{\infty},\left\{\gamma_{n}\right\}_{n=0}^{\infty},\left\{\beta_{n}\right\}_{n=0}^{\infty} \in(0,1)$ satisfy the following conditions:

(i) $0<\delta \leq \delta_{n} \leq \beta_{n} \leq \gamma_{n} \leq \gamma \leq \frac{1-\delta}{1+L^{2}}$;

(ii) $\lim _{n \rightarrow \infty} \delta_{n}=0$ and $\sum_{n=0}^{\infty} \delta_{n}=\infty$.

Then, the sequence $\left\{x_{n}\right\}_{n=0}^{\infty}$ generated by (3.32) weakly and strongly converges to the fixed point of $T$.

Corollary 3.3. Let $H$ be a real Hilbert space, $K$ a nonempty closed convex subset of $H$ and $T: K \longrightarrow K$ is $\alpha$-demicontractive mapping. For any arbitrary $x_{0} \in H$, define the sequence $\left\{x_{n}\right\}_{n=0}^{\infty}$ iteratively as follows:

$$
\left\{\begin{array}{l}
x_{n+1}=P_{K}\left[\left(1-\alpha_{n}-\gamma_{n}\right) x_{n}+\gamma_{n} T y_{n}\right] \\
y_{n}=\left(1-\beta_{n}\right) x_{n}+\beta_{n} T x_{n}, n \geq 1
\end{array}\right.
$$

where the sequences $\left\{\delta_{n}\right\}_{n=0}^{\infty},\left\{\gamma_{n}\right\}_{n=0}^{\infty},\left\{\beta_{n}\right\}_{n=0}^{\infty} \in(0,1)$ satisfy the following conditions:

(i) $0<\delta \leq \delta_{n} \leq \beta_{n} \leq \gamma_{n} \leq \gamma \leq \frac{1-\delta}{1+L^{2}}$;

(ii) $\lim _{n \rightarrow \infty} \delta_{n}=0$ and $\sum_{n=0}^{\infty} \delta_{n}=\infty$.

Then, the sequence $\left\{x_{n}\right\}_{n=0}^{\infty}$ generated by (3.33) weakly and strongly converges to the fixed point of $T$.

Corollary 3.4. Let $H$ be a real Hilbert space, $K$ a nonempty closed convex subset of $H$ and $T: K \longrightarrow K$ is demicontractive mapping. For any arbitrary $x_{0} \in H$, define the sequence $\left\{x_{n}\right\}_{n=0}^{\infty}$ iteratively as follows:

$$
\left\{\begin{array}{l}
x_{n+1}=P_{K}\left[\left(1-\alpha_{n}-\gamma_{n}\right) x_{n}+\gamma_{n} T y_{n}\right] \\
y_{n}=\left(1-\beta_{n}\right) x_{n}+\beta_{n} T x_{n}, n \geq 1
\end{array}\right.
$$

where the sequences $\left\{\delta_{n}\right\}_{n=0}^{\infty},\left\{\gamma_{n}\right\}_{n=0}^{\infty},\left\{\beta_{n}\right\}_{n=0}^{\infty} \in(0,1)$ satisfy the following conditions:

(i) $0<\delta \leq \delta_{n} \leq \beta_{n} \leq \gamma_{n} \leq \gamma \leq \frac{1-\delta}{1+L^{2}}$;

(ii) $\lim _{n \rightarrow \infty} \delta_{n}=0$ and $\sum_{n=0}^{\infty} \delta_{n}=\infty$. 
Then, the sequence $\left\{x_{n}\right\}_{n=0}^{\infty}$ generated by (3.34) weakly and strongly converges to the fixed point of $T$.

Competing Interest. The authors declare that there is no conflict of interest.

\section{References}

[1] F.E. Browder, Nonlinear mappings of nonexpansive and accretive type in Banach spaces, Bull. Amer. Math. Soc. 73 (1967) 875-882.

[2] F.E. Browder, W.V. Petryshyn, Construction of fixed points of nonlinear mappings in Hilbert space, J. Math. Anal. Appl. 20 (1967) 197-228. https://doi.org/10.1016/0022-247X (67)90085-6.

[3] C.E. Chidume, Picards Iteration for nonlinear Lipschitz strong pseudocontractions in uniformly strong Banach spaces, ICTP Preprint, IC/951, (1995) 88. https://www. osti.gov/etdeweb/biblio/194049.

[4] C.E. Chidume, Geometric properties of Banach spaces and nonlinear iterations, Springer-Verlag, London, (2009).

[5] C. Chidume, C. Moore, Fixed point iteration for pseudocontractive maps, Proc. Amer. Math. Soc. 127 (1999) $1163-$ 1170. https://doi.org/10.1090/S0002-9939-99-05050-9.

[6] C.E. Chidume, S.A. Mutangadura, An example of the mann iterative method for lipschitz pseudocontractions, Proc. Amer. Math. Soc. 13 (1974) 2359-2363.

[7] T.L. Hicks, J.D. Kubicek, On the Mann iteration process in a Hilbert space, J. Math. Anal. Appl. 59 (1977) $498-504$. https://doi.org/10.1016/0022-247X(77)90076-2.

[8] N. Hussain, A. Rafiq, M.S. Kang, Iteration schemes for two hemicontractive mappings in arbitrary Banach spaces, Int. J. Math. Anal. 7 (2013) 863-871.

[9] D.I. Igbokwe, Construction of fixed points of strictly pseudocontractive mappings of Browder-Petryshn-type in arbitrary Banach spaces, J. Fixed Point Theory Appl. 4 (2004) 137-147.

[10] E.E. Epuke, Approximation of fixed points and solutions of variational inequalities for certain classes of mappings using hybrid iteration scheme, Unpublished M.Sc. Thesis, University of Nigeria, Nsukka, (2010).

[11] L. Qihou, On Naimpally and Singh's open questions, J. Math. Anal. Appl. 124 (1987) 157-164. https: //doi .org/ 10.1016/0022-247X (87)90031-X.

[12] G. Marino, H.-K. Xu, Weak and strong convergence theorems for strict pseudo-contractions in Hilbert spaces, J. Math. Anal. Appl. 329 (2007) 336-346. https://doi.org/10.1016/j.jmaa. 2006.06.055.

[13] L. Maruster, S. Maruster, Strong convergence of the Mann iteration for $\alpha$-demicontractive mappings, Math. Computer Model. 54 (2011) 2486-2492. https://doi.org/10.1016/j.mcm.2011.06.006.

[14] M.A. Noor, K.I. Noor, T.M. Rassias, Some aspects of variational inequalities, J. Comput. Appl. Math. 47 (1993) 285-312. https://doi.org/10.1016/0377-0427(93)90058- J.

[15] M.O. Osilike, Strong and weak convergence of Ishikawa iteration methods for a class of nonlinear equations, Bull. Korean Math. Soc. 37 (2000) 153-169.

[16] M.O. Osilike, F.O. Isiogugu, Weak and strong convergence theorems for nonspreading-type mappings in Hilbert spaces, Nonlinear Anal.: Theory Methods Appl. 74 (2011) 1814-1822. https://doi.org/10.1016/j.na. 2010. 10.054.

[17] M.O. Osilike, A.C. Onah, Strong convergence of the Ishikawa iteration for Lipschitz $\alpha$-hemicontractive mappings, Ann. West Univ. Timisoara - Math. Computer Sci. 53 (2015) 151-161. https://doi.org/10.1515/ awutm-2015-0008.

[18] L. Wang, An iteration method for nonexpansive mappings in Hilbert spaces, Fixed Point Theory Appl. 2007 (2007) 28619. https://doi.org/10.1155/2007/28619. 
[19] K.K. Tan, H.K. Xu, Approximating fixed points of nonexpansive mappings by the Ishikawa iteration process, J. Math. Anal. Appl. 178 (1993) 301-308. https://doi.org/10.1006/jmaa.1993.1309.

[20] Y. Yao, Y.-C. Liou, G. Marino, A hybrid algorithm for pseudo-contractive mappings, Nonlinear Anal.: Theory Methods Appl. 71 (2009) 4997-5002. https://doi.org/10.1016/j.na.2009.03.075.

[21] H. Zhou, Convergence theorems of fixed points for Lipschitz pseudo-contractions in Hilbert spaces, J. Math. Anal. Appl. 343 (2008) 546-556. https://doi.org/10.1016/j.jmaa.2008.01.045.

[22] H. Zhou, Demiclosedness principle with applications for asymptotically pseudo-contractions in Hilbert spaces, Nonlinear Anal.: Theory Methods Appl. 70 (2009) 3140-3145. https://doi.org/10.1016/j.na. 2008.04.017.

[23] A.B. George, Weak and strong convergence of the Ishikawa iterative sequence to fixed points of Lipschitz pseudocontractive maps in Hilbert spaces, Adv. Fixed Point Theory, 5 (2015) 147-157.

[24] E.I. Veluhan, Weak and strong convergence algorithm for Lipschitz Pseudocontractive Maps in Hilbert Spaces, Unpublished M.Sc. Thesis, Department of Mathematics, University of Nigeria, Nsukka, (2014).

[25] O.O. Owojori, Some convergence results for fixed point of hemicontractive operators in some Banach spaces, Kragujevac J. Math. 31 (2008) 111-129.

[26] C. Morales, J. Jung, Convergence of paths for pseudo-contractive mappings in Banach spaces, Proc. Amer. Math. Soc. 128 (2000) 3411-3419. https://doi.org/10.1090/S0002-9939-00-05573-8.

[27] I. K. Agwu, D. I. Igbokwe, Hybrid-type iteration scheme for approximating fixed point of Lipschitz $\alpha$-hemicontractive mappings, Adv. Fixed Point Theory, 10 (2020) 3. https://doi.org/10.28919/afpt/4442. 